\title{
IMPORTANCIA DE LA VERDAD Y LA MORAL EN LA RESOLUCIÓN DE CONFLICTOS
}

Louis Kriesberg

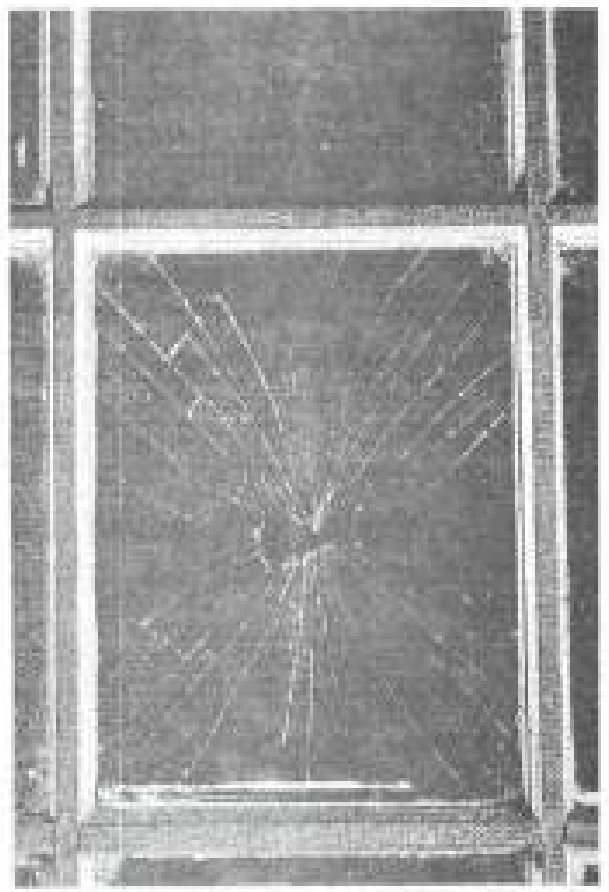

El grupo LNPAX de la Vicerrecuaria te Invexhigativin y

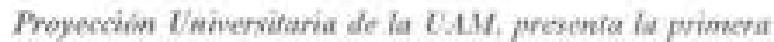
parte dol ensayo escrito por Louis Krievberg sobre la Resolación de Conflictex, con el propesite de continuur publicando escritos que tienen que wer con of tema mencionada

Traduccion del Inglex: I. Oskar L.lane I. Dirretor, Departamenta de Idiemas. Extranjeros. Uimersidad Auteinouna de Manizales

nin manera Je canoterizar has dificaltades descritas por Arthu Koestler ansigte en istablover un equilihos en ef activisme dirigsdo espirituahmeats, pere socialmente inefecivo, como es el easo de un Yogi o de un Comisario que descuida los medion utilizados para logras transformaciones socibeconcimicas deseathies. Fiste es un aspecto que ea motivo de diacusion frocuente; Algones ationea afirman que este es un tema de gran discusión. Aleunos aseguran que kas nccanes bien intereionadas de las personas con frecuexcin conducen a resultados indeneables, mievirns que ta persona que ae halla diapaesta a actuar de manera hrufal venera pranden benefiens. Jeunlmente, otros argumentan que el actuar volentarnente con ef proposito de generm huenes resultsulas es algo que se halla condensdo al fractso y a peoducit efectos nepativos 
Listo argumento tiene muchas implicaciones en el campo de la resolveión de eonflictos. Los mediadorea y los protaponistas enfrentan muchos interrogantes al tratar de tesolver conflictos en banqueda de la justicia, la paz y Is libertad, al tiempo que tratan de evitar muentes, kssiones $y$ atros oostes. ¿Cuàndo es apropiado para un mediador hombardeat un pais en el cual su gobierno está

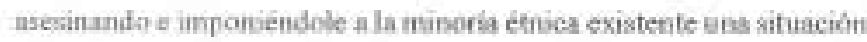
espocial para proteger sus dercehec? ¿Cuảado es apropiado para un inediador el hecho de facilntar el establecimiento de un conflioto entre udverabrios quienes difieren ampliamente en potencia y recursos? Las respucatas varian de acuerdo con los valores y con las cresneras de aquellas que tratan de peoveethas.

Contribuyo con eata diacuaiòn situándota en un contexto más amplie, considerando la nafuraleza y las relactones existentes entre verdad y moral. Ya que tanto la moral y la verdad se contsideran como virtudes, podemos esperar que al ir en la bìiqueda de la una, logremos la otra. En realidad, para algunas personas, en ciertas situsciones, éste es el oaso; poro, frecuentemente, para otras no lo es. Los problemas se identifican mediante el argumento que actabamos de expresar. Con frecucneia la veridad se tevela como inta realidad violenta y la moral alszinas veces se considera en terminos de buenas inteneimes: donde dicha cambinaciós puede generar consecuencias infortunadas. En este documento, examino las contradiceiones posithles que se presentan en la bosiqueda de la verdad y ea la lucha pot actuar moralmente, en relación con la resolucion de conflictos y con el proposita de hallat is manera de onnciliar las costradieciones.

Para aqueflos on formación en sociologia y en otras ciencias sociales, en la dẻcada posterior a la Segunda Guerra Mundial. Ia lucha pou ser ehjetivos y ser libres al elegir sus valores constituian objetivos importuntes. Al mismo fiempo, muchos de nosotros entramos en el mundo de las cienicias sociales con el propósito de mejorar la condición humana y compartimos un entendimiento sobre que condiciones requieren un mejoramiegto, como fue el caso de terminar con el racismo y desviar la ameoaza de una gisertil nuclest. Dado nuestro entendimiento de la nafuraleza de In verdad y de como deseubritis y, dado nuestro entendimiento de la moral y de la manera para svanzat hacia ells, necesaramente nos verwos eonfrontados con varios diemas - La tension existente entre trutar de ballar la verdad y ackuar moralmente no se ba reaseito sín y. peot aùn, wuchas personas manifiestan muy poca

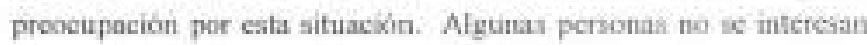
por ella porque dodan de la posibilidad de lograt uno a otro objetivo. o piensan que las dos situaciones son thin diferentes que la una no afecta la ctrn, o creen que son tan ambiguas que fícilracnic pueferi ser compatibles. Sin embargo, ereo que la oxnciliaciòn en la basaqueda de ambos objetivos continûn sisendo un hecho imporianfe que merece mucha ateación, particslarmente en la medidu en que se ielacioua con la resolución de conflictos, Adenàs, el pensarnicnta melectuat reciente $y$ las experiencias ean nuevas politicas pueden contribuir a mejorar nuestro entendimiento de la verbad y de la moral en la resolución de cosflictos y ayudarnos a oxociliar algunos aipectos resullantes de sus contradicciones.

\section{CONCEPTOS BÁsICOS}

La discusion en lo atinente a la verdad, la moral y la resolución de conflictos conidera cada concepto de una manera tnuy amplia. Por eonsiguiente, la verdad puede ser visienta, por ejenjola. de tal mamera que las diferencias de poder sean ganites y que el inas foerte pueda imponer su voluntad, pero puede tambiti ser henigna, enene eit el easo en que las personas compartan identidades y sirnpaticen entre si. Lu moral incluye la busqueda en el avanee de la justicia socal, tal comis ae enfatiza en este documento, peto tambièn ineluye la promocién de la piedad y el olvido, la estabilidad asatenible y ef orden, al tiempo que se evite la erueldaul. Ia resolucion de conaflictos tambien se considera de muscta muy amplia.

\section{PERSPECTIVAS TRADICIONALES}

Ia verdad, considerada tradicionalmeste, constituye la desicripción precisa de la realidad, ae define convo el enteridimiento correcto de lo que ha ocurrido en el pasado y lo que está sucectiendo en la uctualidad Lo anterior supone que la realidad exisfe independientenesite te los observadores y que puede sor aprohondida por cliks. No we pacue conocer is verdad ahsoluta, pero ef sooncieniento es acumatativo y los unvestigstores pusden, de esta mmera, acetearse hacia una vision esda vez ntals preeisa de la verdad.

Tradicionalmente, la büsçueda de la verded requicre de fa confianzan 
Ell metodas reproducibles ųue permitan recolectar y analizar infrimasción. La husqueda debe evitar los sesgos y las proferencias personales. Ni las valores personales ni las expectativas respecto a las consecuencias de ecaceer la verdad tienen que afectar su büsqueda Aàn si no se paeden evitar todos fos seagos y fodas las expectativas subjetivas, se dele tener mucho eaidado para minimizar las distorsiones generadas por la verdad objetiva

La moral. de otra parte, se basa tradicionalmente en las preferencins de los valores y, las prefereneias de los valores no puoden derivarse de las croencias que se tienen sobre Ia realidad In mornl be halla articulada en forma de declaraciones descahies, no bajo la forma de declaraciones factuales. Ejerce autonidad. mediante el entendiatiento compartido, por ejemplo, sobre Diois o la naturaleza humana. Para muchos cientificos sociules, esto ha significado que la moral, a diferencia de la verdad, es cuestión de te y de conviceion, eonstruida socialmonte. La moralidad varia eon les eständares de cads eultura y no puede juzgarsa meduante estandares absolutos.

El sceptar las diferencias existentes entre el cimpo de los valored y la maral y el eampo de los hechos y de la verdad no guiere decir que la gente visi solamente en un campo. La gente necesariamente vive y actúa en amber campos. La büsqueda del progreso boen sea ca lo referente a la verdad o a la moral, con poeo interis en uno $u$ otro aspecto, puede obstacializar el progreso del otro, Los pelieros gencradas por tal pensumienito simple se pueden obocrvar de manera levere. Ën los cipuientes imperativos monales, la información paede distorsionarse, algunas veces de mancra intencional cuando no ae etta en guandia respocio a loe sespos debidos a las expectativas basadas en preferencias. La destorsión tarnbiéa poede ser intencional, cuando la informaciòn ae reoblecta de maneca selectiva y se comunica con ef propesito de adelantar el curso de scción determinado como inoralinento correcto.

De igual manera, casndo se busea la verdad con poco interóa en las situaciones morales se pueden saerificar los derochos humanos, coeno también, cuanda éstos sopa fratados como objetos y son sometides a

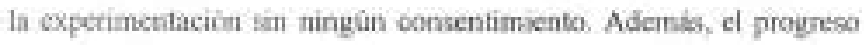
en et cosocimiento puede suministrar instrumentes de eocreión y de oonitrul, $y$ aquellos que poseen el poder para aplicatos lo barain de esa manera, incrementando la explocación de fos demás.

\section{NUEVOS DESARROLLOS Y CONCEPTOS CAMBIANTES}

Las enenepciones tradicionales mobre la verdad y la mocal han aido sujetas a criticas severas. Las peripectivas más recientes merecen vopisideración ya que contribuyen a disminuir alquras de las fensiones existerites entre of avance conjunto de ta verdad y la moralidad. La existencia de la verifad como ente separable de la observación de ella mesma, unu hapòtesis existente en las conxepcionen tradicionales sobre la verdad, estâ siendo implitamente cuestionsda en la actualidad. Lo que sabemos dete derivarse de la observición y debe sonxeterse al filtro de puestros aontidos, no obstanie, Jou issirumenton to magnifiquen. Se deduee que la reabidad poede conooerse solamente bajo eondiciones especificas de observacion. La realidad, por consiguiente, varia segin las condiciones y segù has perapectivas situadas de thanera diversa. Esto ae significa que podames construir la realidad como nosotros qqueramos, las situacioner varian de acuerdo con la potencia de sus predisposiciones respecto ix la manera de ser percihida. Alganas sinuaciones se feconocto ampliamente y de mancra simular y aon eonsistentes con muchas claves de obrervaciones

La interpretsción de les eventos necesariamente depende del marso de referencia del análisis utilizado. Por cjemplo, las comsocuencias de utilizar un medio particular de locha depeaden ampliamente de bas paràmetros de tiempo y de espneio social eonsiderabos. Se pueden sonsiderar las onosecuencias en términos te los efectos inanedalos que se preoontan en el transcunso de las dias is de las efoctos retantados 6 a largo plaw que ie presentan a iraves de las dibedas. Se paeden tamhiēn considerar las cunsecuencias comvo limitadas solanente por aquellos eventos directamente comprometidos en la lacha o por aquellos incluidos solsmente como observadatos, peto que ban recibido algun impsicto

La experiencia y el pensumiento aocial rectentes han ejercido su influencin en el entendimiento setual de la moral. Un desarrollo unportante ha sido el sentido ereciente en lo reforente a que ciertas formas do conducta son casi universaimente depókradas. Aùn aquellas personas que cometen actos eondenados frecuentemeble se escionden o niegan que pertenceen o que son miembros de los grupos que cometicron dichos scins, o las presentan como, otra ciase de actios, pero algunas vees reconcen que su grupo estabs equivocado o que ellos mismos se equivocaton. Lo anteriof ae paiede observaf, a mantera 
de ejemplo, en las declaraciones y acciones que tienen que ver one el Racismo puesueven practica por las blancos de la Repüblica de Sarafrica durante la deceada de 1990. Se puede notar la extensión de las normas compartidas en la seeptación crecieate de la existencia de tos derechos bumansis universales $y$ en la cosidean de los actos úc genocidio, El estudio de los reginànea noermativo en las relaciones intemacionales tamben revela la existencia de catindares monales que tienen influencia en la cosducta de fos gobiernos que los comparten.

La stenicuón prestada a los estándares normstivos cempartidos provee unta base ampita para los imperativos mocales: Esto ke ejemplifica mediante ef argurnento para ef coovencionalismn como la base etica en lns relacionea internacionales y ove otros campos. La Eitica se basa en pruncipios que las personas utilizan para justificar y ganar aceptación por was aeciones por parte de otras. Para ser efectivas, las partes involucradas deben eocnpartir los principios. Mas que promulgar easlęuier tradición etica, particular como la base de una teoria monal. de acuerdo ean esta perspectiva, la obligación momal puede ser y se basa ca el aouerdo respocto a "ciertns reglas consederadas como autarizadas y eiertas practicas censideradas como legitimak, cualquier aspectas que las partes isvolecradas aeverden considerar comn justa o legitims os juste o legitimo". E analisis presentado ex este ensaye se tasa en el enfoque ecovencionalista, sin determinar que existe un vóbigo monal universalmente aceptado $y$ sin afirmar que un deterninade sódigo moral tiene el caracter de supremo. Pero lingupo de los argumentoa presentades en este documento pressapone que cada código moral convencional es igualmente convincente.

Fuera de las nvarsecs en el pessamiento intelectual, has aurgido nuevas peripectivas ormo consecuencia de la aplicación de las politicas sociales eon el proposito de promover el bienestar comùn. Las provisiones gubernamentales y religinsas tendientes a controlar la conducta humana hat existido desde que apareciefon los gobiernos y las instituciones religiosas, La inefectividad frecuente de lsa potiticas en su intento de mejorar la conducta humass y los impactos frecuentes no intencionados $\mathrm{e}$ indescados generados por tales politicas tansbien han sido reconocidos cine el transcurso del fempo. En lasdbeadas recientes. sill entbargi, se he incretnentado el usp del cooocimicata cientifico social con el joposite de contubuit a la formulación de politicas sociales, pero no se ha asegurndo ģue dichas politicas bayan generndo Ins efectra deseados y evitado aquellos indeseados. La evidencia demuestru que las fouenas àmtenciones no parastizas buenos resultados
Unu reapuesta puede ser la del escopticismo que se expetinienta cuando se trata de implementaf ealevier imperativo moral, otra coesiske en examinar muy cuidadocamente las ponsectencias yeneradas por positicas alternas. La precocupacioin respecto a las consecucheias derivadas de tales politicas determina las consideracionies pricticas y resales sobre la moral.

El trabajo en la solución de problerais que tiencen qque ver oon la resolución de conflietos, de manera particulat, the estimulado a los practicantes y analistas a reflexionas mobe in naturileza de la verdad y de la moral. La resolución de tales conflictos haed dirigur In btencién hacia las interpetacioces sambiantes tunio presentes cromo pasadas censtrusdas por las paries en contlicto, aun sotre kos mismos evenos. Ademds, la intrespeeción de muchos practicantes de la no violencia $y$ de la resolución de confficios indica que a traxes de la imvestagacien matua tiodas las partes pueden tener an ascesen mais completo a la verdad. La exploración se presenta de muchas formas, tales coma aquellas presentes en el contextio de los taileren inferactivos of de las confrontaciones durante una campañล no violenta

L. a vesplasón de contlictos se tama de ina mainera ampés en oste ensayd.

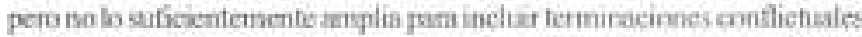

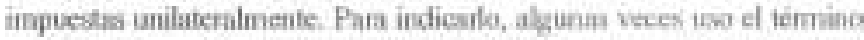
solución de problemas que tienen que ver eson la resplacién de conflictos

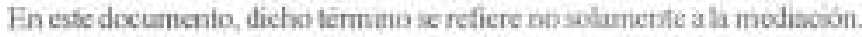
o a la tezociación, mas bien. comprende las fornas contruativas detitro de las cuales se deben conducir lok erentlictros, incluyendo la luchs no viokenta que trata de fransformar les oponentes en asicisdoe pan la selución de problemss. Comprende tambiên las politieas que tiender a desviar las lechas destrictivas, la transfomacton de los conflictos destruativos y apareniemente insolubles on conflictos solubles.o ain en una relación colaborativa entre partes reconciliadas que anterioemente eman enchigas.

La experiencia reaultante de las actividades teodientea a ta resolucjèn de contlictos requiere que se fe preste atención a las sétunciones éticas. Es cierto que los nediadores y utra clase de participantes enfrentan opciones que involueran preooupseinees morales rospecto al derecho Ue intervención y de como intervenif. Adeonás. las partes envalukrades en In lutha se esfuerzesn en justificar motahnente suk aceiones ante sus cogartidaring y aliados, al igual que ante sus sdversarios. Crando las purtes en contlieto adoptan un enfoque parm la fesolocion de sus 
oonflictos, las stuaciones morales se tornan particularmente importantes. Una posicion tamada poit algunas personas precicupsdas por la moral respexto a las varias formas de resnlver eonflevtos consiste en asumir las necessidailes bumanas taisicas o en Jeterminar los valoners hatsicas particulares on principion monales que deberian guiar el trabsio tendiente a la reapluciòn del conflicto. James Laue y Gictald Cormick. por ejemplo, afirman que is cticu de la resolucioún de conflictos se basa en "la premisa bísica que las personas 800 inheresteinente valsosas y que debon ser tratadas cono fines en si mismas": 34. Ellog derivan tes valores: fundumentales de esta premisa: el empoderamiento proporsional. Ia jesticia y la blertad, y con base en dichoc valores derivan varios principios éticos a ser seguidos por los participantes. Aunque fichas difeotivis puedan ser muy ótiles, pueden be ser aceplatas por las partes en tenflicto, o las vanas partes en conflicto o los participantes les pueden asignar ana priondad diferente a dichos valores y principios

La roflexiôn sohre los conilictos evidencia el hochn de que riugsin medio de loch y ninguma solución posec cualidades buenas o malas ne mezcludas. Cauls cuno de aceson enearna una mezula de caracteristieas morales. Por cjamplo, las petsunas pooden estar livetuando poe kaym un futuro con mejor justicia social. peri al hacerio reducen la likertud de muches $y$ te involuerali en bafos de sangre y experimentan grandes perdidas en d presente; o una soluciòn pucde terminar eon el baño Ae senere, pete scamente do tuna mansers beve y de al forma que genere nuevas injusticias. E1 insistir-ell la primacia del propio orfonamieoto axiológico y de los propíos principion morales parece entrar en contradiccián con algunos aspectos de la perspectiva referente a la solueion de conflictos.

\section{ENFoQues cLínicos $\mathbf{Y}$ UNIVERSALES}

Se pueden aplicar los conceplos de verdad y de mocal a eventos especifices of se pueden utilizar con referencis a todas las combunidades o a ciertas categoriah de persanas en circunstancias especificas. Et primer uso refeja un enfoque clínico y ef ultimo, un enfoque generatieantic.

Las personas que trabajan en el hreal de resoluciob de conflictos frecuentemente se preoceran por um conflicto especifioo y por lo que hay que hacer al respecto. El imodelo médien puede ser îtil para el cntendimiento de las implicaciones consecuentes de tal kafasas Los mbtiens generalmeste valoran sus habilidades clinucas: Ia habilidad para extablocer un dingnóstice respecto a lo que to esta sucediendo a un paciente en particular en un tiempo determinado, Is habilidad para decifit cual es la terapia más deotive parn tratar dicho paciente. Peto an lss interaceiones peculiares y anicas entre muchos procesas gescrales las que responden por el estado o condecíón de cala paciente. De ofra parte, Jos cientificos que estudian una enferrnedad expecifies tratan de ecoocer solve las diferentes condiciones y prosesos que interaetuas eson el proposita de expliear las variaciones que ae peesentan en el curso de tal entermedad. Su vonocimiente se enmates generalmente ea términos de probabilidades, Para los legisladores en et campo de la salud, tales probabilidades constituyen una base ravonable para determinar las politicas tendienter a evitar lss enfermedades.

En realidaul ambos cufoques solit descables. Se requiere mucha información para tratar un paciente. La sabjduria y la experiencia requeridas para aplicar teorias generales a un cass especifico que se halla buju consideracobots tienen un eariacter invaluable, Ia sinderesis constituye tambico un elemento crucial. Is geseralización a partir do eutas experiencias parn situarlas on un universos mayor, peode verse cargada con grandes riesgos. La manern de adquirir encoscimiento universal depende de otron métodus.

La verdad, entances, puede téferirse a lo que conocemos y entendemon respocto in una persons o confficto en particular o a lo que sabernos respocto a los procusks generales de lit vids secat y de ke conflictos sociales. De igual manera, la moral puede refenise a lo que es correcto hacer en una circunstancia particular o a fo que es generalmente earrecto en la mayoria de las cireunatancias. En este ensayo; utilizo ambes enfoques. Fl lector debe reconneer que las observaciones, desde un punto de vista, no son necesarianente lss misenas si se founan desde otra perspectiva.

En la prictica de la resslucion de conflictos, el cnfoque climico es preeminente Loa solucionadores de conflictos utiloan sus estándures morales y sacan sus propiua eonclusiones respeeto a la yerdad con el propósito de preacribir acciones para los adveraatios y para ellos mismars an sus luetsa particulares. Los analistas en resoloción de conflictos algunas veces -3ambien se sitisfaceri eon la preseripción de politicas, aplicando $5 u s$ onnocimientus sobre verfader y aspectoa moralea rolntivamente generales a 
easos especifiess. Tumbica pueden proveer prescripciones geverales pura las diferentes cluses de conflicios, pero mury frocuentemente inberpiztan eafterzos passdos aplicados a la tesoluciòn de conflictos I la laz. de enterpretaciones gerierales sobre ta vendud $y$ is moral.

\section{CONTRADICCIONES QUE SE PRESENTAN EN LA BÚSQUEDA DE LA VERDAD Y DE LA MORAL}

Aun coe estas concepeiones implias sobre la yerdad y solve it moral, Ia busqueda de una de clas pucde, algunas vees, inierferir con el avanee de la otra. En consecuencia, de aiguna manera la busapeda de la verdail pucte resultar en lo que podrit ser visto, de manern amplia, eomo una ponducta immoral. En realidad, muchas personas deplorun alganos de loc efector de has insovanones tecnologicas con base en el avanoe de urs verdad por pante de los cientifious nanimles, bocn sean armss nuckeares. perticidas o uutomovilea. En verdad, la mayorit de estas desamollat tecnclagions tiener efectrs difusos, machos de los cuales pasan desuperahidowo son indescablex, no obstank, muchos de dichos cfoctos has sido aquelles desados por las eolectividades. Diehos efectos we yen influenciados por las estructuras sociales y por las celturas, at igat geve poe las politicas seciales, enn base es los grades variables. de eonceimienta social. La bisqueda de la verdad respecto a las situscionies sociales por parte de lns cicntificos sociales, sin emsharvo. eonstituye un punto do importancia escneial en auestro casa.

Aumque ta fooris ylos metidos de las ciesctias seciales no han producido la clase de poder qoes se etseuentri en las ciencias naturales. la información y las tecnicas de las ctencias sociales provecit recursos que las comunidules puedeny, en realidad, emplean en el bogo de sus objetivos. Lè anterior se manifiesta en el mereadeo de prodicsos, en la bisqueda de votas durante las campaîas electorales, en la buscqueda de apoyo prara polificas scovides particulares y en la movilizacion se grupos para Iitear batallas of park apoyar un establecimienko paciboo. Gran parte de la informución y de low sećtrodow de las ciencias suciales se utiliza más ficil y más efectivanente por acuelias polectividades con ventwigs

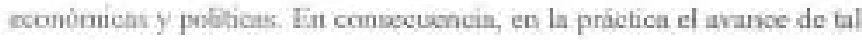
bonocimiento wo tiene un carieter neutral. Erevuentemente fortaleve a squellos qoe 300 dominantes destro del sistema social establecido y. ademis, poenciulmente ohstaculizan los esfuerzos que condukes a la generacion de cambios cou el propósito de redacir las desigualdades:
De otra parte, las ciensiss sociales pueden nocavir la autoridad de los dominastes, hecho que tunbien puede considerarse cono contrario at la moral. Mucha parte do la vidia social se halla sostenida y constreniida por mitos de crieen común y de solidaridad, por la confiamea en los. logros últimos de la justicia y por crecacias particulares respecto a la naturaleza humana. Lia busqueda de la verdad respecto a tales satuaciones frecuentemente torna la fortma de desmitificación y de

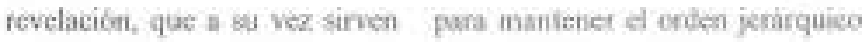
exintepte. Tal revelaciòn puede entonces tehilinur ol prodch mocal

En el campo de la resolución de confictos safgen muchas contradicxiones entre la verdad y la moral. Los mediadores tratan de centribuir a In moral promoviendo el establecimiersto de un acuerdo entre los antagonistas que confronmen varias coutradicetones fos metiadores, en general, pueden ereer en ef vaise de la honestad, al menos con el propousito de retener la eresthilidad con fos advermans, pero, con frecuencia, ponen en practea algan gsudo de decopeica an la expectativa de que ae alennzasta dicho acoerdot, La decopenie puede presentarse mediante el informe selectivo whre lo que una parts piensa acerea de los térmanos que debe aceptar, o puede prescritarye

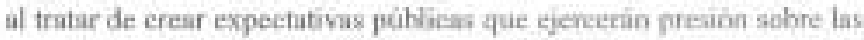
partes en confliets

Las contradieciones también pureecn surgir de la estructura inibereate de in felacion existente entre los adverwarios, heehe que afecta a los tmismes adversanos y a los posabies intemnediarios. Por cjemplo, la asimetria que se presentain en los recursos disponibies paza cada parte en oonflioto en lo referente a la verdad tiende a moldear low términos de un acuerdos pero, con f́recuencia. et la parte inenos favorecida la que en realidad merexe un redifescionamiento. Los intermediarios debes entonces optar por actuar de tal manera que contribuvan a un objetivo moral măs que a otro, por ejciशplo, bien sẹ

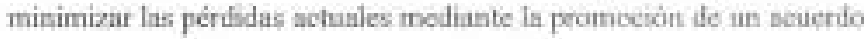
rapido o maximizar la justicia futuma mednate el fortalecimacato de fo parte más debal y prolongar, de eana manera, la lucha. Se poede fẹducii el difemu por medio de creencias en la probabilidad de Jograr realmente, uno o otro objetivo: In probahilidad percibidu puede favorecer fucrtemente la adopción de una nifernativin ea hagar de olra. En un mundo en el cual tas persoeas croent que lis fiecza coetcitiva constituye el arbitro máximo de los conflictos sociales; el apaciguamiento de un adversario podes verke como una invitacion a la geresien o aceptar de manera insoral un restitado no satisfactorio. 
La buaqueda $y$ la conefianza en la verdad pueden pareeer meralmeme contradietotias debodo a la complejiéad de los conflictos socialé. Las díspatas a pran exala involucran machas purfes y muchas situacionew. Bien sea mediante el anafnis, ol seguimiente o la busquolli de he resolusion de an conflicto, se dehen tociar las deciseones con base en in mayar importancia de algunas partes y

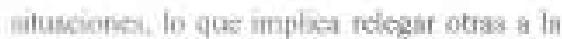
periferia. Tre inevo, In creencias que tienen In persumar respecte a Is posibilidad de futuros deaurnollios afectan fas opeiones que toman las personas para hacer to que consideran eteco. Por onensigunente, al esperar que sna parte sea intransigente, en un conificto con varias partes, las otras partes cmeprometidas con ef conflicto actuarin de mancti desituctiva ai no se llega màpidamenic a un acuedo para resolverlo y escluiran la parte que cansideran intransigente. Liths intereses de la farte coulabila se verain rendidos. tal cumo sucedie cene el pobiemo bntimico y el sobiernie frances en la situación can Choccolivaqua en 1938, sn so fallido interiso por apaciguar la Alemana Nazi Las accinnei han eide condentadas ampliamente como munocules y tambítr convo encheat en alpunas de sas pinemisas 357.427 . De otra parte, en el cabo de la Cress Cubima en Cetubre de 1962 los jefou de estado a soviótico y americanc exhiyerot a Cuha de sas negoctaciones en at intento por reseher la crises. Dudos los riespos Donsecusater de la prolangacion de la crisisy quiris del hocho de set incapacer de resolveris. ell esac easo fa exclusoćn de ania perte oun una participación amplia en el conflicto fue exsarderada monalinentic cocrova

Finalmente, obaervo cono cl tratur de avanzar en la trasqueda de la moral pasde interferit eno la busquesta de la verdad. El companiso ero ciertre principios morales poede inducir a his personas a evitar el eximent o a roconocer verdates que eonsederan pueden sceavar u obotaculicar lin implementicion de dichos primeipios. En el campo do lon conflictos unciales, par cjemplo, el becho de eatar involucrado con fa rectitod de is distribacide o con la rodencion del perdins puede ejereer alguna intluenca on la evideneia que se busca y que se mabys. De iganl manent, la lealtad con ciertas jentidaden menores tales cono una comunidad etrios especifiea of la lealed con identidades mov ampitias que uncluyea fos adversanow afecta las luchas y la maneza compo se evaluan, bien wea como perdidas 0 como ganancias. Log compromesos con la moral tendientes a evitar masacrsi o a poner en riesgo la vida de las personas de una comunidad pueder también canalizar la aeleceion de la evidencia y la botabdad de las agentas do imvestigacion

\section{LA MUTUALIDAD EN EL AVANCE DE LA VERDAD $Y$ de La MORAL.}

Haste el mousenlo, of fectoe habea perisado en muchos deusvierdes son bo gae he eserito y habna periado en les formas màs pertinentes para fortalescr la verdad y la monal, las cuales no son incompatibles entre si y que, en efecto, pueden ser Gtiles mutumente. Me dediearé ahoni a discuatif toino estas Kusquedas poeder apoyarse mutuanente.

\section{CONTRIBUCIONES DE LA VERDAD AL AVANCE DE LA MORAL.}

Relativamente muy pocas nersonas ven la moral couno el simple becho de actuar corrictamente y no us procxupan. de mameta primaria, por fax consecueneiss de sus aeciones, mas bien tratan de expresat as convicciones.y do daries sentido, La muyoria de las porsonas, op obstante, se proocipan por las efectas de sas accionied y tratan ic producir lo que elion esossiderai cono buegis efectoa morules. Hasta tusto las emnecuencias de la conitueta motal sean consideradas de importancia, el cotvocumiento $\mathrm{de}$ la vendad le avudu a la moral a avaarar en varias tormas.

Tal como se andico en la discusaon antenor sobre el eonocimento clinicu y senber la toora general, la verdad poede pertenecer a domasios vunanter qoe van dende atuaciones localen e hatóricamente expecificas hasta fonomenos universales y genenalec. Para la mayoris de las persinas, el actuar moralmente th aun of decidit solate ha que es moralmeate correcto depende, de manera parcial, de las consecuencias anticipadan rexulianies de accionen particulares, II comicumicato acceca de las covaceuencias posibles ise lasa, hasta cierto puisto, ell la infonmación detallada respecto a lax peculiandades de uns situación cspecifica. Cuanto mejor sea la información $y$ la introspeecibil que se tiene de un contilicto en particular o de eualquera situacion, muyen Is porabitidad de seleccirutiar politicas que el pubblico en general considera estàn de acuerds son la inoral. En tonsesusicia, se facilita ertances in selección entre varion imperativa! unomies, algumas voces itoonsistentes.

La vendad se fefiefe a proposiceines tecincas geocrales y a generalizaciones empiriess que proveen informacion útal en el momento de decidir a que pruscipo momal se le debe dat mayor priortad de scuerto con las 
circunatancias. Lus proposicinnes teóricas y las generalizaciones empinicas necesanatnetile tionden a set abstractsa y no pueden, por si nismss, justificar acciones particulares en ef marce de conflicks particulares. No obstante, pueden indiear probabilidades de desarrolloe que apoyariant ciertas clases de conducta mass que otras

Además, dos areas comprendidas en la busqueds de In serdid tienen una importanscia trascendental para actuar moralmente. Dea es la súsqueda def conceimicnto respecto a bos sentimientos morales que los seres humanos comparten cusando se ballan en comunidad. Dicho ecoecimiento provec Ins belges para estructurar formss construetivas tendientes a actuar frente a las luchas y a resolver oonflictos, ya que lo pueden suministrar a los adversarion que se ballan en dicbas comuaidades, la base comùn para limitar el comportamiente destructivo y hallar un resultado reciprocarsente aceptabie.

La otra àrea que es motivo de investigacion importante es aquella que se dirige busaa al deseubnmiento de las bases para obsener resultados relativamente morales y consobidar otra actividades socialea. Como analistas de conflictus, quicoes los resuet ven, y los protagenistas, necesilames conover mís acercu de las circunatanoias y los procesos que generati tana conducta moral efeetiva, tomo tambièn un somportamiente inmoenl destrostive. Por cossiguieate, no sobamente netesilams campresder eomo se experimentan y como se cjecutan las politicas a faveir del genocidio, ino también como evitarlas y enfrentarial. Par cjemplo, los andiliais de las tasas de sobrevivientes Judios es kai paises que estuvieron bajo la seupación nazi y las telactones de comumidades o srupos particulares que ayudaron o protegieron a las Judies y a otros objetivos de bs politicas genocidales de los Nazis sumiaistran una información invalaable la cual contribuye Al avanec do le moral

La tratsformaciòn de les confliotos destructivos, con granden exmponentes de conducta innonal, en luchas ounstructivas constituye frecueatemente el producto de muches pasos determinados por muchas persona o comunidades, Aûn los puatos de eneueatro dramáticos y repentinos son, con frecuericia, el resultado de eambios previos goe han aido construidos Ientumente por la presión y el upoyo de aqueflos eventos que han sido considerailos como criticos y transformadoses. Por consigiente, mochas peraonas puoden y en reabdad contribuzen a tales transformaciones de formas variables pero no inconsecuentes. De ipual manera, ef tratamiento de confliclos enastruetivos y el logro de resultados onsstructivos, que minimicen la destructividad inmoral, requieren de is labor de muelas personas

\section{REFERENCIAS}

BERLIN. Isaish (1998) "My Inteliectual Futh". The New York Review of Books; $53-60$

BURTON, Jehn (1990). Cosfliset: Resolution and Prevention. Ners York: St. Martin's

ERIKSON, Eink H. (1969), Gandhi's Truth. New York: W.W Norton

FEIN, Heien (1979). Acounting for Genocide National Responses and Jewish Vietimization During the Holocaust, New Yock: The Free Press:

ASHER, Rneald (1997). Interactive Conflict Resolution. Syracuse Syracuse University Press.

GOI.AN, Mafti (1976). The Sectet Conversations, af Henry Kisainger: Step-by-Sicp Diplomacy in the Middle East. New York: Bantum

HALLIF, Philip (1979). Lest Innocent Blood Be Shed. New York Harper \& Row.

KOESTL SK. Arthur (1967). The Yogi and the Comunissar and Other Essays. New Yotk: MeMillan.

KRASNER. Stephan (1983). International Revimes. Ithaca, N. Comell University Press

KRukSBERG, Louis (1989). Poaoe: Meaningi, Polatiea, Strutegjes New Yorki Pracket/Greenwood.

NADER, Laura (1991) Harmany Models and the Construction of Law. Now York: Greenwood Press 